\title{
Ralstonia solanacearum fatty acid composition is determined by interaction of two 3-ketoacyl-acyl carrier protein reductases encoded on separate replicons
}

Sai-Xiang Feng ${ }^{1 \dagger}$, Jin-Cheng $\mathrm{Ma}^{1+}$, Ji Yang ${ }^{1}$, Zhe Hu${ }^{1}$, Lei Zhu ${ }^{2}$, Hong-Kai Bi ${ }^{3}$, Yi-Rong Sun ${ }^{4}$ and Hai-Hong Wang ${ }^{1 *}$

\begin{abstract}
Background: FabG is the only known enzyme that catalyzes reduction of the 3-ketoacyl-ACP intermediates of bacterial fatty acid synthetic pathways. However, there are two Ralstonia solanacearum genes, RSc1052 (fabG1) and RSp0359 (fabG2), annotated as encoding putative 3-ketoacyl-ACP reductases. Both FabG homologues possess the conserved catalytic triad and the N-terminal cofactor binding sequence of the short chain dehydrogenase/reductase (SDR) family. Thus, it seems reasonable to hypothesize that RsfabG1 and RsfabG2 both encode functional 3-ketoacyl-ACP reductases and play important roles in $R$. solanacearum fatty acid synthesis and growth.
\end{abstract}

Methods: Complementation of Escherichia coli fabG temperature-sensitive mutant with $R$. solanacearum fabGs encoded plasmids was carried out to test the function of RsfabGs in fatty acid biosynthesis. RsFabGs proteins were purified by nickel chelate chromatography and fatty acid biosynthetic reaction was reconstituted to investigate the 3-ketoacyl-ACP reductase activity of RsFabGs in vitro. Disruption of both RsfabG genes was done via DNA homologous recombination to test the function of both RsfabG in vivo. And more we also carried out pathogenicity tests on tomato plants using RsfabG mutant strains.

Results: We report that expression of either of the two proteins (RsFabG1 and RsFabG2) restores growth of the E. coli fabG temperature-sensitive mutant CL104 under non-permissive conditions. In vitro assays demonstrate that both proteins restore fatty acid synthetic ability to extracts of the E. coli strain. The RsfabG1 gene carried on the $R$. solanacearum chromosome is essential for growth of the bacterium, as is the case for fabG in E. coli. In contrast, the null mutant strain with the megaplasmid-encoded RsfabG2 gene is viable but has a fatty acid composition that differs significantly from that of the wild type strain. Our study also shows that RsFabG2 plays a role in adaptation to high salt concentration and low pH, and in pathogenesis of disease in tomato plants.

Conclusion: $R$. solanacearum encodes two 3-ketoacyl-ACP reductases that both have functions in fatty acid synthesis. We supply the first evidence that, like other enzymes in the bacterial fatty acid biosynthetic pathway, one bacterium may simultaneously possess two or more 3-oxoacyl-ACP reductase isozymes.

Keywords: $R$. solanacearum, 3-ketoacyl-ACP reductase, Type II fatty acid synthase system

\footnotetext{
* Correspondence: wanghh36@scau.edu.cn

${ }^{\dagger}$ Equal contributors

'Guangdong Provincial Key Laboratory of Protein Function and Regulation in Agricultural Organisms, College of Life Sciences, South China Agricultural University, No.483 Wushan Road, Tianhe, Guangzhou 510642, P. R. China Full list of author information is available at the end of the article
}

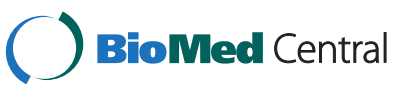

(c) 2015 Feng et al. Open Access This article is distributed under the terms of the Creative Commons Attribution 4.0 International License (http://creativecommons.org/licenses/by/4.0/, which permits unrestricted use, distribution, and reproduction in any medium, provided you give appropriate credit to the original author(s) and the source, provide a link to the Creative Commons license, and indicate if changes were made. The Creative Commons Public Domain Dedication waiver (http://creativecommons.org/publicdomain/zero/1.0/) applies to the data made available in this article, unless otherwise stated. 


\section{Background}

Fatty acid biosynthesis is essential for the survival of mammals, plants, fungi and bacteria $[1,2]$. Using acetyl-CoA and malonyl-CoA as the initiating substrate and building block, respectively, these organisms share a common set of biochemical reactions to extend fatty acyl chains by two carbon atoms per cycle [1-3]. In most bacteria and plants, fatty acids are synthesized by a discrete and highly conserved group of enzymes designated the Type II or dissociated fatty acid synthase (FAS) system [2-5]. As a key feature of the FAS II system, the hydrophobic fatty acyl intermediates are shuttled from enzyme to enzyme by a small, highly acidic acyl carrier protein (ACP) [3, 4]. To date, the FAS II system has been most extensively studied in the Escherichia coli model system, in which all the enzymes required for fatty acid synthesis have been identified and characterized biochemically [2-4].

The genes encoding fatty acid synthetic enzymes are highly conserved in bacteria, and in many cases, their genomic arrangement is also conserved [2, 3, 6]. Although the basic steps in the fatty acid synthesis cycle are common to all bacteria [3, 4], abundant exceptions to the $E$. coli paradigm are present in other bacteria $[4,7,8]$. In general, a discrete enzyme encoded by a single chromosomal locus catalyzes each step of the elongation pathway. However, although some bacteria encode isozymes, in most cases these enzymes have differing specificities [7, 8]. One example is Enterococcus faecalis, which encodes two homologues each of FabZ and FabF. However, this bacterium uses one of the FabZ homologues (now called FabN) and one of the FabF homologues (now called FabO) to perform the unsaturated fatty acid synthetic functions performed by E. coli FabA and FabB $[9,10]$.

Another example is the enzyme enoyl-ACP reductase, which catalyzes the NAD (P) $\mathrm{H}$-dependent reduction of the enoyl-ACP double bond in the last step of the elongation cycle [7]. Several bacteria have two enoyl-ACP reductases that can be either of the same or of different protein families [11-13]. In some cases one of the enzymes is responsible for supporting a fatty acid synthetic rate that allows wild type growth. The rationale for this duplication of enzyme activity is unknown, but the two enzymes of a given bacterium often differ in their inhibition by triclosan, a man-made biocide [11-14].

In the bacterial fatty acid synthesis pathways studied to date, only a single enzyme, FabG, has been found to catalyze the reduction of 3-ketoacyl-ACPs to 3-hydroxy acyl-ACPs [2, 3] (Fig. 1a). FabG proteins are particularly difficult to annotate because they are members of the short-chain dehydrogenase/reductase (SDR) protein superfamily, which constitutes one of the largest protein superfamilies, with many bacterial members [15, 16] (Fig. 1b). Hence, annotation of a gene as encoding a 3-ketoacyl$\mathrm{ACP}$ reductase is much more likely to be accurate if the gene is located within a cluster of genes that are good candidates for having roles in fatty acid synthesis. That said, our prior work showed that only one of the two Lactococcus lactis annotated fabG genes was involved in fatty acid synthesis, although both genes had plausible genome contexts [17].

We report the first example of a bacterium that encodes two functional FabG homologues. This bacterium is Ralstonia solanacearum, a soil-borne, destructive plant pathogen that has a global distribution and an unusually wide host range [18]. Functional characterization of the two proteins shows that they both are active in fatty acid synthesis but play different roles in determining the cellular fatty acid profile, the response to environmental stress, and pathogenesis.

\section{Results}

Two $R$. solanacearum genes annotated as fabG homologues

Two $R$. solanacearum genes, called fabG1 and fabG2 (RsfabG1 and RsfabG2 in this study), were annotated as encoding homologues of E. coli FabG, the essential 3ketoacyl-ACP reductase [18]. The RsfabG1 gene (RSc1052) is located in a chromosomal cluster of putative fatty acid synthesis genes $(f a b H, f a b D, a c p P$ and $f a b F)$, based on alignments of their gene products with the E. coli proteins, whereas the RsfabG2 gene (RSp0359) lies within a second putative fatty acid synthesis gene cluster $(f a b B, f a b F 2$ and $K A S$ ) located on the megaplasmid (Fig. 1c). Sequence alignments indicated that RsFabG1 and RsFabG2 are $65 \%$ and $43 \%$ identical to E. coli FabG, respectively, and showed that the catalytically active short chain dehydrogenase/reductase (SDR) family triad (Ser, Tyr and Lys) and the N-terminal cofactor binding sequence (Gly motif [GlyXXXGlyXGly]) defined by the X-ray crystal structures of E. coli FabG [16, 19,20 ] are present in both $R$. solanacearum proteins (Fig. 1b). Moreover, two E. coli FabG residues, Arg-129 and Arg-172, reported to play critical roles in facilitating the binding of the ACP moiety of the substrate [21], are conserved in RsFabG1 and RsFabG2 (Fig. 1b). Based on these criteria, it seemed reasonable to hypothesize that RsfabG1 and RsfabG2 both encode functional 3-ketoacylACP reductases and that both play important roles in fatty acid synthesis and growth in $R$. solanacearum.

\section{Complementation of an $E$. coli fabG temperature-sensitive mutant with the RsfabG genes}

E. coli strain CL104 is a fabG temperature-sensitive mutant [22] that lacks 3-ketoacyl-ACP reductase activity at $42{ }^{\circ} \mathrm{C}$ and is unable to grow at this non-permissive temperature. To test the functionality of RsFabG1 and RsFabG2 in vivo, each of the genes was inserted into the arabinose-inducible vector pBAD24M [11] to give the expression constructs pYJ3 (RsfabG1) and pYJ4 (RsfabG2). 
A

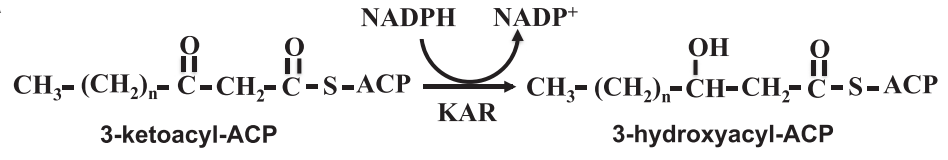

B
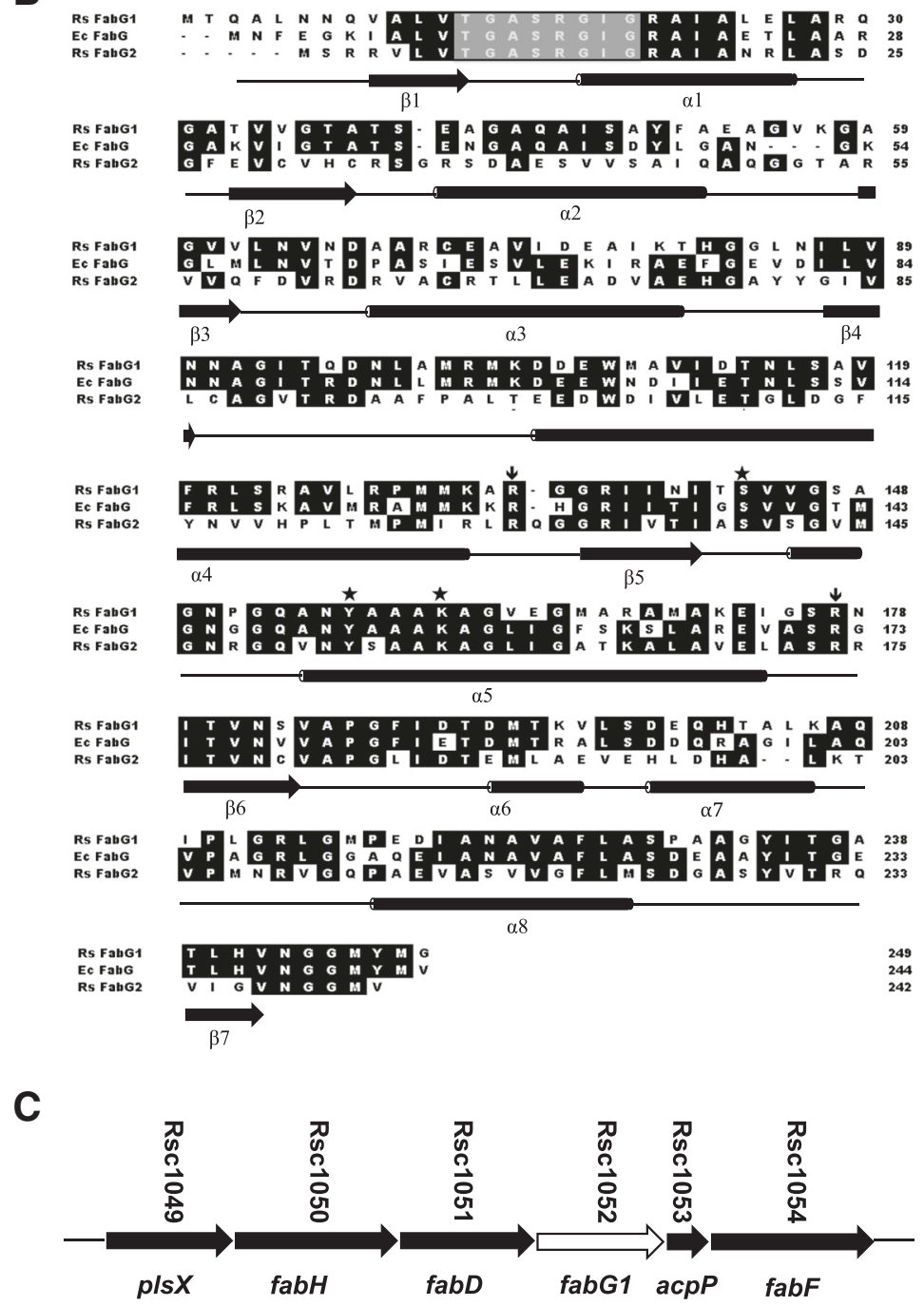

Chromosome

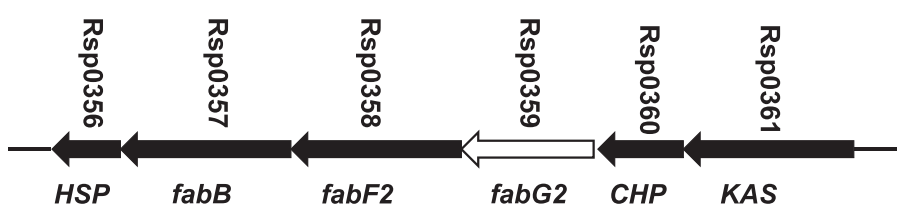

Megaplasmid 
(See figure on previous page.)

Fig. 1 The 3-ketoacyl-ACP reductase (KAR) reaction, alignment of $R$. solanacearum FabG1 and FabG2 with E. coli FabG and organization of the $R$. solanacearum fatty acid biosynthesis gene clusters. Panel $\mathbf{a}$, The KAR reaction. Panel b. Alignments of $R$. solanacearum FabG1 and FabG2 with E. coli FabG. Rs and Ec denote R. solanacearum and E. coli, respectively. The cofactor binding sequence (Gly motif [GlyXXXGlyXGly]) is boxed. The two arginine residues thought to bind the ACP moiety are highlighted by arrows and the catalytic triad residues are highlighted by asterisks. The alignment was done with Clustal W based on identical residues. Panel c, Organization of the $R$. solanacearum fatty acid biosynthesis gene clusters. The thick arrows indicate the relative sizes of the genes. The numbers above the arrows indicate the gene designations in the $R$. solanacearum of Comprehensive Microbial Resource (CMR) database, and the gene names below the arrows indicate the $E$. coli genes that correspond to the open reading frames in the R. solanacearum cluster. HSP indicates hypothetical signal peptide protein. CHP indicates conserved hypothetical protein. KAS indicates putative

3-ketoacyl-acyl carrier protein synthase

The plasmids were transferred into strain CL104 at the permissive temperature and the resulting transformants were tested for their growth at the non-permissive temperature. Strain CL104 carrying plasmid pYJ3 grew at $42{ }^{\circ} \mathrm{C}$ even in the absence of arabinose (Fig. 2). In contrast, the pYJ4containing strain grew at $42{ }^{\circ} \mathrm{C}$ in the presence of arabinose but failed to grow in the absence of arabinose. Therefore, both of the RsFabG candidates could complement the $E$. coli fabG(ts) strain, indicating that each protein catalyzes 3ketoacyl-ACP reduction. However, it appears likely that RsFabG2 is less active than RsFabG1 because high-level induction by arabinose was required for robust growth.

\section{Expression and purification of the $R$. solanacearum FabGs}

To perform a direct in vitro assay of FabG activity, recombinant $\mathrm{N}$-terminal hexahistidine-tagged RsFabGs were produced. These proteins were purified by nickel chelate chromatography to obtain preparations that gave single bands on SDS-gel electrophoresis (Fig. 3a). The purified RsFabG1 and RsFabG2 proteins have monomeric molecular weights of $29 \mathrm{kDa}$ and $30 \mathrm{kDa}$, respectively. Given that E. coli FabG is a homo-tetramer [20], we estimated the solution structures of RsFabG1 and RsFabG2 by gel filtration chromatography (Fig. 3b). The RsFabG1 and RsFabG2 elution profiles showed that both FabGs, like E. coli FabG, exist as homo-tetramers in solution.

To elucidate the secondary structure and folding properties of the FabG protein, circular dichroism (CD) spectroscopy analyses were used (Fig. 3c). The CD spectrum of $R$. solanacearum FabG1, like that of E. coli FabG, had the characteristic helix signature with minima at 208 and $222 \mathrm{~nm}$, whereas FabG2 showed only a single helix signature minimum at $222 \mathrm{~nm}$. Moreover, the helical content of RsFabG2 (22.5 \%) was considerably lower than that of EcFabG (33.2 \%), whereas the helical content of the RsFabG1 (30.9\%) was much closer to that of EcFabG. These data indicated that, although both RsFabG1 and RsFabG2 are homo-tetramers in solution, they seem likely to have different folding patterns.

\section{In vitro enzymatic activities of RsFabG1 and RsFabG2}

The function of the two RsFabGs in fatty acid synthesis was assayed in vitro. First, the enzymatic activity of RsFabGs in the initial steps of fatty acid synthesis was tested as described in the Methods. In the absence of a 3-ketoacyl-ACP reductase, only holo-ACP was seen (Fig. 4a, lane 1), probably due to hydrolysis of the labile 3-ketobutyryl-ACP during electrophoresis [23]. Addition of RsFabG1, RsFabG2 or EcFabG to the reaction mixture resulted in production of butyryl-ACP (Fig. 4a, lanes 2-4). Upon addition of the long-chain E. coli 3-ketoacyl-ACP synthase, EcFabB, to the reactions, all reactions produced long-chain acyl-ACP species (Fig. 4a, lanes 5-7). These data clearly showed that, like E. coli FabG, both RsFabG1 and RsFabG2 could complete the initial cycle of fatty acid synthesis to produce butyryl-ACP.

Next, the enzymatic activity of the two RsFabGs in the reduction of long-chain 3-ketoacyl-ACP substrates was also examined. Incubation of EcFabB with malonyl-ACP and octanoyl-ACP or tetradecanoyl-ACP resulted in the formation of 3-ketodecanoyl-ACP (Fig. 4b, lane 2) or 3-

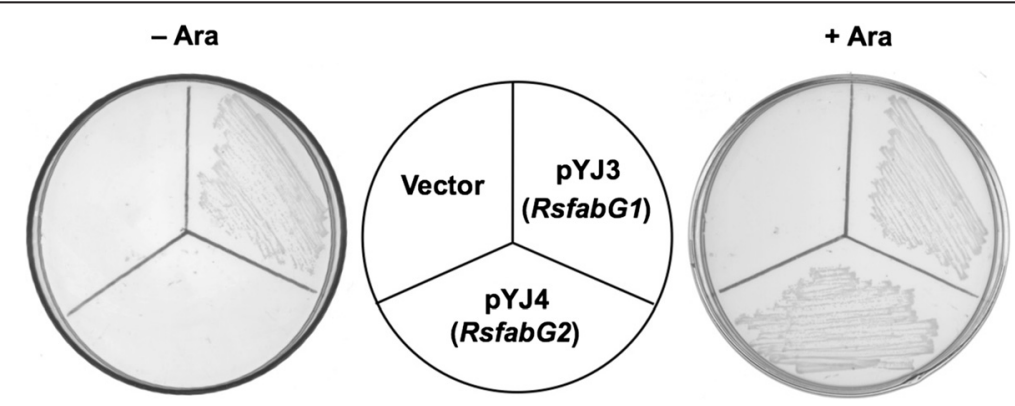

Fig. 2 Growth of transformants of E. coli fabG(Ts) mutant strain CL104 with plasmids carrying the R. solanacearum fabGs. E. coli strain CL104, carrying the pBAD24M-derived plasmids pYJ3 (RsfabG1) and pYJ4 (RsfabG2), was grown at $42^{\circ} \mathrm{C}$. ARA, abbreviation of arabinose 
A

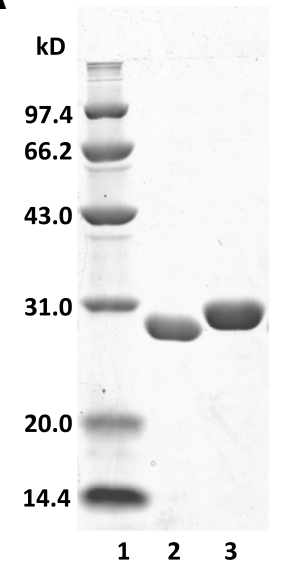

B

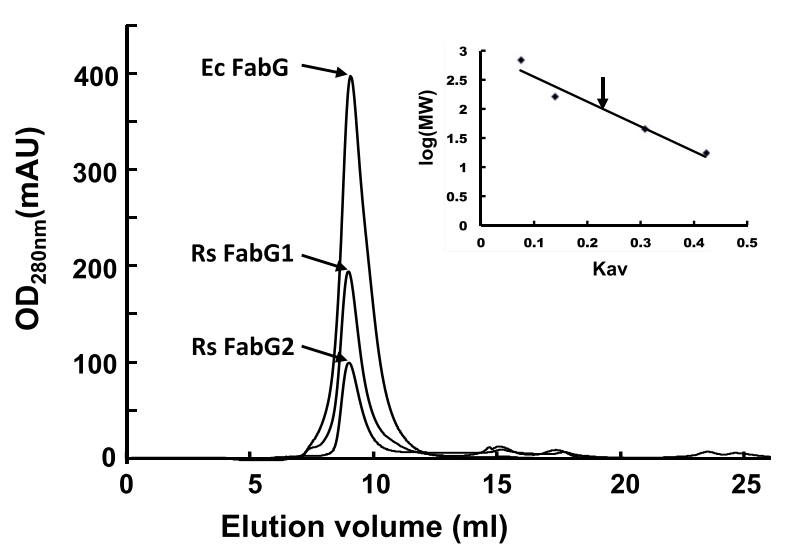

C

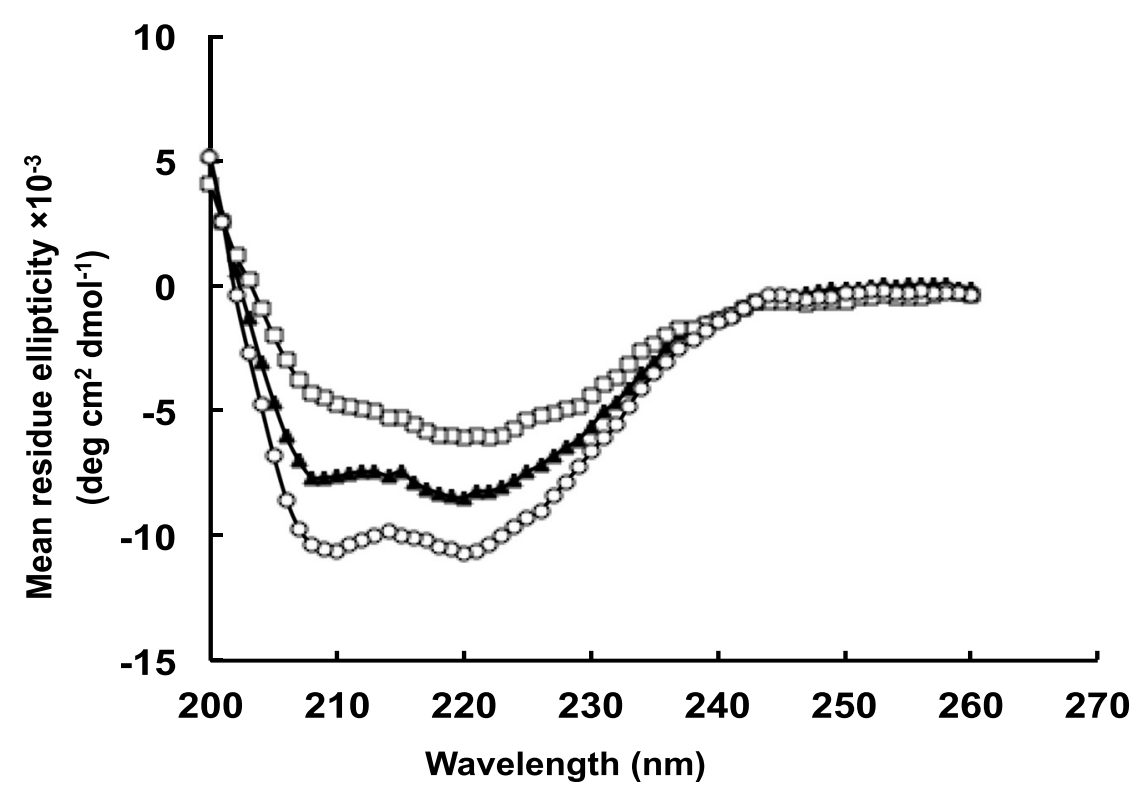

Fig. 3 Purification of R. solanacearum FabGs from E. coli strain BL21 (DE3) and circular dichroism spectra of R. solanacearum FabG1 and FabG2. Panel a, Purification of R. solanacearum GMI1000 FabG1 and FabG2 by native nickel-chelate chromatography. Lane 1, molecular mass markers; lane 2, R. solanacearum FabG1 protein; lane 3, R. solanacearum FabG2 protein. Panel b. Size exclusion chromatography of the hexahistidine-tagged wild type RsFabG1 and FabG2 protein. The elution peaks of the molecular weight standards are given at the top of the panel. Panel $\mathbf{c}, C D$ spectra of R. solanacearum FabGs. CD spectra were measured at $25^{\circ} \mathrm{C}$. Open circles, E. coli FabG; filled triangle, $R$. solanacearum FabG1; open squares, $R$. solanacearum FabG2

ketohexadecanoyl-ACP (Fig. 4c, lane 8), respectively (the longer-chain species are more stable during electrophoresis). Upon the addition of NADPH, a 3-ketoacyl-ACP reductase (EcFabG, RsFabG1 or RsFabG2) and EcFabZ to the reaction mixture all incubations produced 3hydroxyacyl-and enoyl-ACP species (Fig. 4b, lanes 3-5, and c lanes 9-11). Note that EcFabG converts 3-ketoacylACPs to 3-hydroxacyl-ACPs, whereas E. coli FabZ dehydrates 3-hydroxacyl-ACPs to produce enoyl-ACPs. Therefore, these data indicate that both RsFabGs are active with long-chain 3-ketoacyl-ACP substrates, consistent with their ability to support the growth of E. coli.

Acetoacetyl-CoA is the substrate most often used to test 3-ketoacyl-ACP reductase activity (the cognate acyl-CoA substrate often functions in place of the acyl-ACP substrates although generally with a much higher $\mathrm{Km}$ value) [16]. Thus, the kinetic properties of RsFabGs (Table 1) were examined using the model substrate acetoacetylCoA as substrate. RsFabG1 reduced acetoacetyl-CoA, although with a lower enzyme activity than E. coli FabG, 
A

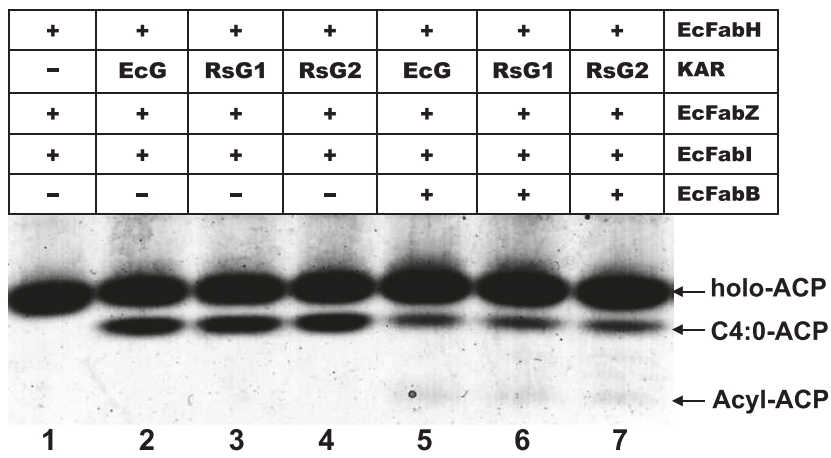

B

\begin{tabular}{c|c|c|c|l|}
+ & + & + & + & EcFabB \\
\hline- & EcG & RsG1 & RsG2 & KAR \\
\hline- & + & + & + & EcFabZ \\
\hline
\end{tabular}

halo-ACP $\rightarrow$

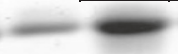

$\mathrm{C} 8: 0-\mathrm{ACP} \rightarrow=$

trans-2-C10:1-ACP $\rightarrow$

1

23

345

56

C

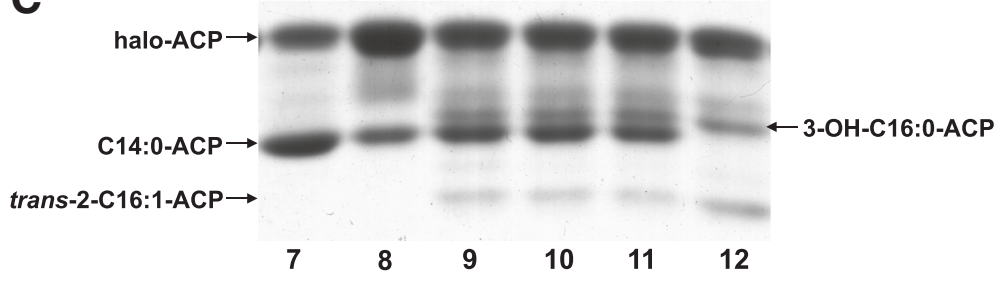

Fig. 4 Function of $R$. solanacearum FabG1 and FabG2 in fatty acid synthesis reactions. Panel a, Function of R. solanacearum FabG1 and FabG2 in the initial cycle of fatty acid synthesis. The initial cycle of fatty acid synthesis was reconstructed in vitro using a combination of E. coli FabH (ECFabH), KAR (E. coli FabG (EcG) (lane 2), or R. solanacearum FabG1 (RsG1) (lane 3) and FabG2 (RsG2) (lane 4), E. coli FabZ (EcFabZ) and Fabl (EcFabl) enzymes, ACP, $\mathrm{NADH}$, and NADPH as cofactors and malonyl-ACP plus acetyl-CoA as substrates to produce butyryl-ACP. To complete the fatty acid synthesis reaction (lanes 5 to 7), E. coli FabB (ECFabB) was added to the reactions (the faint bands are due to the instability of short-chain 3-ketoacyl-ACPs in the electrophoresis gels). Panel $\mathrm{b}$ and $\mathrm{c}$, Function of $R$. solanacearum FabG1 and FabG2 in the fatty acid elongation cycle. The elongation reaction mixture contained E. coli FabB (EcFabB), KAR E. coli FabG (EcG) (panel b, lane 3 or panel c, lane 9) or R. solanacearum FabG1 (RsG1) (panel b, lane 4 or panel c, lane 10), FabG2 (RsG2) (panel b, lane 5 or panel $\mathbf{c}$, lane 11) and E. coli FabZ (EcFabZ), malonyl-ACP plus octanoyl-ACP (panel b) or tetradecanoyl-ACP (panel c) as substrates, and NADH and NADPH as cofactors. RsG1 and RsG2 denote R. solanacearum FabG1 and FabG2, respectively. Lane 1 is the product of octanoyl-ACP and lane 7 is the product of tetradecanoyl-ACP. Lane 6 is the product of 3-hydroxydecanoyl-ACP treated with E. coli FabZ and lane 12 is the product of 3-hydroxyhexadecanoyl-ACP treated with E. coli FabZ

Table 1 Kinetic parameters of R. solanacearum FabG proteins

\begin{tabular}{|c|c|c|c|c|c|c|}
\hline & \multicolumn{2}{|c|}{$V_{\max }(\mathrm{nmol} / \mathrm{min} \times \mu \mathrm{g})$} & \multicolumn{2}{|l|}{$\mathrm{K}_{\mathrm{m}}(\mu \mathrm{mol} / \mathrm{L})$} & \multicolumn{2}{|l|}{$\mathrm{K}_{\text {cat }}\left(\sec ^{-1}\right)$} \\
\hline & $\overline{A A C^{a}}$ & $\mathrm{NADPH}$ & $\mathrm{AAC}^{a}$ & $\mathrm{NADPH}$ & $\overline{A A C}$ & NADPH \\
\hline EcFabG & $97.02 \pm 8.5$ & $3.54 \pm 0.5$ & $4372.6 \pm 105.1$ & $5.41 \pm 1.3$ & $45.28 \pm 4.3$ & $1.65 \pm 0.43$ \\
\hline RsFabG1 & $4.70 \pm 1.3$ & $1.50 \pm 0.5$ & $313.28 \pm 14.5$ & $25.75 \pm 3.8$ & $2.19 \pm 078$ & $0.71 \pm 0.24$ \\
\hline RsFabG2 & $N D^{b}$ & ND & ND & ND & ND & ND \\
\hline
\end{tabular}

${ }^{a}$ AAC denotes acetoacetyl-CoA

${ }^{b} \mathrm{ND}$, could not be detected 
whereas RsFabG2 failed to reduce the model substrate (Table 1). The maximal velocities of acetoacetyl-CoA reduction of RsFabG1 or EcFabG were strikingly different, as were the Km values of RsFabG1 and EcFabG. The maximal velocity of RsFabG1 for the NADPH substrate was lower than that of EcFabG, and the Km of RsFabG1was higher than that of EcFabG (Table 1).

\section{Essentiality of the $R$. solanacearum RsfabG1 gene}

To examine whether the two 3-ketoacyl-ACP reductases are essential for $R$. solanacearum cell growth and cellular fatty acid synthesis we attempted disruption of both genes. Plasmids containing pK18mobsacB-borne Gm resistance cassette insertions in RsfabG1 (pYJ27) or RsfabG2 (pYJ30) were constructed. Plasmids (pYJ27 or pYJ30) carrying the gene disruptions were introduced into the genome of wild type $R$. solanacearum strain GMI1000 via conjugal transfer from $E$. coli S17-1. Mutant colonies were subsequently screened on a medium containing sucrose and gentamicin. The success of the mutants was assayed by colony PCR analysis using two primer pairs listed in Additional file 1: Table S2. During our attempts to generate an RsfabG1-disrupted $R$. solanacearum strain, only single crossover integrants were obtained (Additional file 2: Figure S1, A). This result suggested that RsfabG1 is an essential $R$. solanacearum gene.

To test this hypothesis we constructed plasmid pYJ33, which carries RsfabG1 under the control of the E. coli lac promoter, and transformed this plasmid into an RsfabG1 single crossover integrant stain (Additional file 2: Figure S1, A). The transformants were then plated on medium containing gentamicin, sucrose and IPTG to select for loss of $s a c B$ function. Colony PCR assays using the primer pair RsFabG1 upside and RsFabG1 downside showed that one of the surviving colonies, named strain RS-G5 (RsfabG1::Gm/pYJ33), in which RsfabG1 was replaced by the gentamicin resistance gene, contained the defined $2.0 \mathrm{~kb} \mathrm{Gm}$ resistancecontaining fragment. As expected, a $2.3 \mathrm{~kb}$ RsfabG1-containing fragment was amplified from the wild type strain using the same primer pair (Additional file 3: Figure S2, A). Using primer pair RsFabG1ck1 and RsFabG1ck2, colony PCR amplified a $0.7 \mathrm{~kb}$ RsfabG1-containing fragment from both the wild type and RS-G5 (RsfabG1::Gm/pYJ33) strains because Rs-G5 (RsfabG1::Gm/pYJ33) contains the plasmid-borne pYJ33 copy of RsfabG1 (Additional file 3: Figure S2, B). Insertion of the $\mathrm{Gm}$ cassette plus deletion of RsfabG1 sequences was also confirmed by sequencing of the PCR fragment of the disruption allele.

Moreover, we constructed pYJ32, a pK18mobsacBborne plasmid containing E. coli fabG plus a downstream Gm resistance cassette. Plasmid pYJ32 was transformed into the wild type strain GMI1000 and the transformants were processed through the counter-selection protocol described above (Additional file 2: Figure S1, B). This gave strain RS-G3 (RsfabG1::EcfabG) in which RsfabG1 had been replaced by E. coli fabG as shown by colony PCR analysis (Additional file 3: Figure S2, A and B) with same primer pairs as used above (Additional file 1: Table S2).

Next, the strains lacking a functional chromosomal copy of RsfabG1, including RS-G5 (fabG1::Gm/pYJ33) and RS-G3 (fabG1::EcfabG), were tested for growth in M63 medium at $30{ }^{\circ} \mathrm{C}$. Under IPTG induction, strain RS-G5 (fabG1::Gm/pYJ33) grew in M63 medium, although it had a generation time of $256 \pm 10$ min which is appreciably longer than that of the wild type $R$. solanacearum strain GMI1000 (150 $\pm 7 \mathrm{~min})$, but RS-G5 (fabG1::Gm/pYJ33) failed to grow without IPTG induction. However, the E. coli FabG substitution strain RSG3 (RsfabG1::EcfabG), which had a generation time of $157 \pm 4 \mathrm{~min}$, grew as well as the wild type strain. Collectively, these data demonstrated that RsfabG1 is an essential gene in $R$. solanacearum and that its function can be replaced by the E. coli fabG gene.

As assayed by gas chromatography-mass spectrometry (GC-MS), though both mutants contained the same fatty acid species as the wild type strain on M63 medium plates, the two mutant strains all produced more unsaturated fatty acids (UFAs) and fewer 3-hydroxy fatty acids (3-HFAs) than the wild type strain GMI1000 (Table 2). The ratios of UFAs to SFAs in strains RS-G3 ( $f a b G 1:: E c f a b G$ ), and RS-G5 (fabG1::Gm/pYJ33) were 1.09 and 1.29, respectively, much higher than that of the wild type strain (0.57). In addition, the ratios of 3-hydroxy fatty acids to non-hydroxyl fatty acids in these strains were 0.35 , and 0.44 , respectively, lower than that of the wild type strain (0.66).

\section{Effects of RsfabG2 mutation on growth of $R$. solanacearum}

Using a similar approach, we readily obtained a RsfabG2 Gm insertion mutant (named strain RS-G2 (RsfabG2::Gm)) (Additional file 2: Figure S1, C). The growth of Rs-G2 (fabG2::Gm) in M63 medium was tested. In M63 medium at $30{ }^{\circ} \mathrm{C}$, Rs-G2 (RsfabG2::Gm) had a generation time of $170 \pm 5 \mathrm{~min}$, somewhat longer than that of the wild type strain (150 $\pm 7 \mathrm{~min}$ ), indicating that RsfabG2 was not essential for $R$. solanacearum growth.

The GC-MS assays showed that strain Rs-G2 (RsfabG2:: Gm) also contained significantly more UFAs and fewer 3-HFAs than the wild type strain (the UFA/SFA ratio for Rs-G2 (RsfabG2::Gm) was 1.16; the HFA/FA ratio was 0.57) (Table 2). However, in the RS-G2 strain the major increase in UFAs was in $C_{18: 1}$ species, whereas both $\mathrm{C}_{16: 1}$ and $\mathrm{C}_{18: 1}$ species of UFA were increased in RsfabG1 mutant strains (RS-G3 and RS-G5). This suggested that, like RsFabG1, RsFabG2 plays a role in determining the cellular fatty acid profile of $R$. solanacearum and that 
Table 2 Fatty acid composition of total lipid extracts from $R$. solanacearum fabG mutant strains grown on M63 medium plates ${ }^{a}$

\begin{tabular}{lllll}
\hline Fatty acid & RS-G2 (fabG2::Gm) & RS-G3 (fabG1:EcfabG) & RS-G5 (fabG1:Gm/pYJ33) & GMl1000 \\
\hline n-C14:0 & $2.32 \pm 0.09$ & $3.00 \pm 0.10$ & $2.99 \pm 0.13$ & $2.38 \pm 0.40$ \\
n-C14:0 3-OH & $32.53 \pm 2.24$ & $21.49 \pm 0.28$ & $29.68 \pm 2.54$ & $39.00 \pm 2.87$ \\
n-C16:1 cis 9 & $11.54 \pm 1.16$ & $16.88 \pm 0.48$ & $20.82 \pm 1.50$ & $10.52 \pm 1.11$ \\
n-C16:0 & $23.25 \pm 1.25$ & $28.71 \pm 1.94$ & $22.76 \pm 1.75$ & $28.47 \pm 3.74$ \\
n-C16:0 2-OH & $2.14 \pm 0.44$ & $1.59 \pm 0.09$ & $1.03 \pm 0.12$ & $2.06 \pm 0.16$ \\
n-C17:0 cyclo & $5.76 \pm 0.73$ & $5.51 \pm 0.74$ & $1.14 \pm 0.24$ & $1.89 \pm 0.17$ \\
n-C18:1 cis 11 & $17.20 \pm 2.43$ & $17.37 \pm 2.12$ & $16.96 \pm 0.67$ & $8.98 \pm 0.96$ \\
n-C18:0 & $4.21 \pm 0.36$ & $4.62 \pm 0.29$ & $4.31 \pm 0.29$ & $6.54 \pm 0.89$ \\
UFA/SFA & 1.16 & 1.09 & 1.29 & 0.57 \\
HFA/FA & 0.57 & 0.35 & 0.44 & 0.70 \\
\hline
\end{tabular}

${ }^{a}$ Cells were grown on M63 plates at $30{ }^{\circ} \mathrm{C}$ for two days. The total lipids were extracted and transesterified to obtain fatty acid methyl esters, and products were identified by GC-MS. The values are the means \pm standard deviations of three independent experiments and the percentages of total fatty acids

${ }^{b}$ UFA denotes unsaturated fatty acid, including C16:1 cis 9, C17:0 cyclo and C18:1 cis 11. SFA denotes saturated fatty acid, including C14:0, C16:0 and C18:0. HFA denotes hydroxyl-fatty acid, including $\mathrm{C} 14: 03-\mathrm{OH}$ and $\mathrm{C} 16: 02-\mathrm{OH}$

some changes in 3-ketoacyl-ACP reductases affect the fatty acid composition of $R$. solanacearum.

In order to investigate possible physiological functions of RsFabG2, we also tested the growth of strain RS-G2 (RsfabG2::Gm) in BG medium under various environmental challenges (high temperature, high salt concentration or low $\mathrm{pH}$ ). Although the growth of strain RS-G2 (RsfabG2::Gm) was similar to that of the wild type strain at different temperatures (data not shown), high salt concentration $(0.1 \mathrm{M} \mathrm{NaCl})$ or low $\mathrm{pH}$ (pH 5.5) differentially affected the growth of strain RS-G2 (RsfabG2:: Gm) (Fig. $5 \mathrm{~b}$ and $\mathrm{c}$ ). Note that under natural conditions $(\mathrm{pH} 7.0$ without addition of $\mathrm{NaCl}$ ) all strains grew well (Fig. 5a). Under these conditions, RS-G2 (RsfabG2::Gm) grew more slowly than the wild strain GMI1000 (Fig. 5b and c). Complementation of RS-G2 (RsfabG2::Gm) with

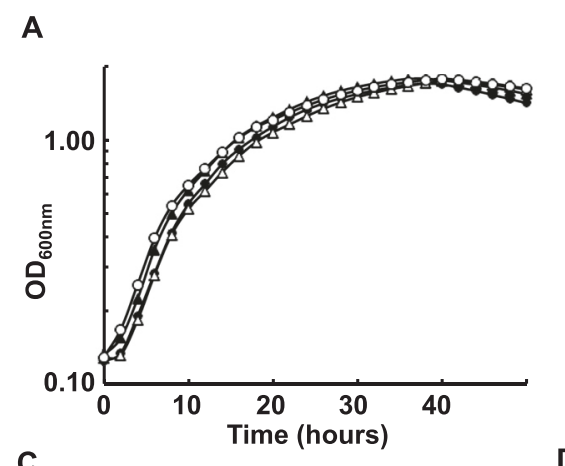

B

C

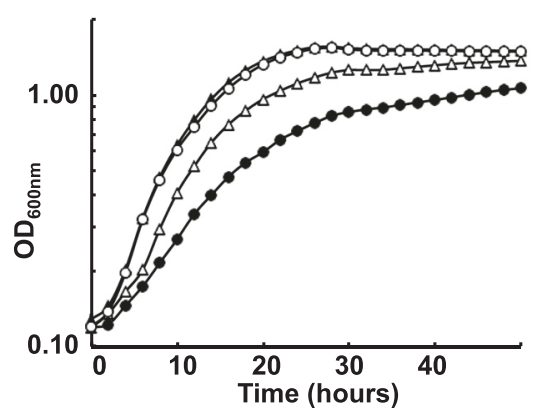

D
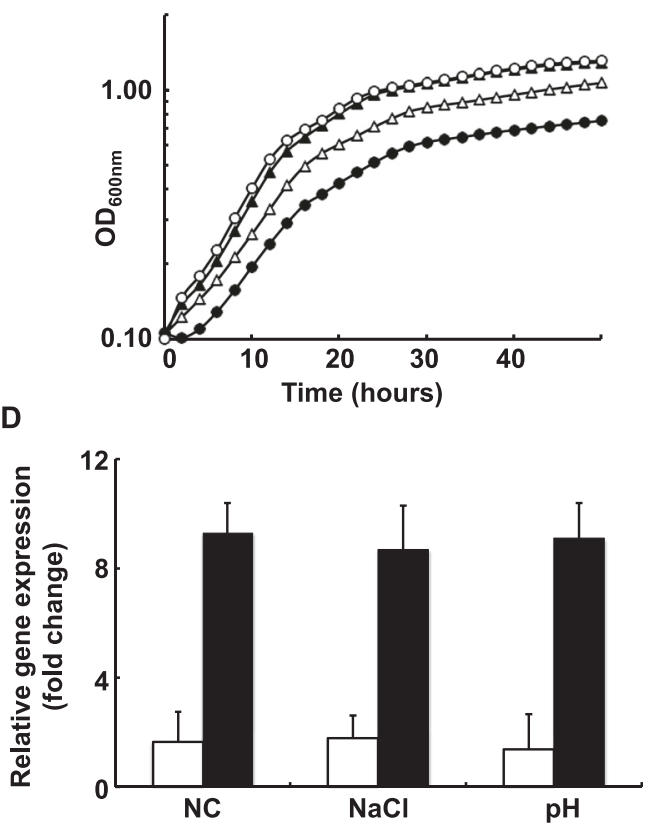

Fig. 5 Growth of the R. solanacearum fabG2 mutant strain in BG medium under high salt or low pH conditions. Panel a, The growth of mutant strain RS-G2 in BG medium under natural conditions $\left(30^{\circ} \mathrm{C}, \mathrm{pH} 7.0\right.$ and no addition of $\left.\mathrm{NaCl}\right)$. Panel $\mathbf{b}$, The growth of RS-G2 in $\mathrm{BG}$ medium containing $0.1 \mathrm{M}$ $\mathrm{NaCl}$ at $30^{\circ} \mathrm{C}$. Panel $\mathbf{c}$, The growth of RS-G2 in BG medium with pH adjusted to 5.5 by $1 \mathrm{~N} \mathrm{HCl}$ at $30^{\circ} \mathrm{C}$. Filled circle denotes RS-G2 strain, empty triangle denotes RS-G2/pYJ34 strain, filled triangle denotes RS-G2/pYJ35 strain and empty circle denotes GMI1000 strain. Panel d, Expression of RsfabG1 and RsfabG2 under hight salt or low pH conditions. Open bar denotes RsfabG1 and filled black bar denotes RsfabG2. 
plasmid pYJ34, which carries wild type RsfabG2, restored the growth of RS-G2 (RsfabG2::Gm) (Fig. 5). These results indicated that, although RsfabG2 is not an essential gene in $R$. solanacearum, it does play a role in the adaptation of $R$. solanacearum to environmental stress. To test whether the expression of RsfabG2 is controlled by environmental stress, the transcript level of RsfabG1 and RsfabG2 was examined under high salt concentration and low pH by quantitative RT-PCR (Fig. 5d). The level of expression of RsfabG2 was 6- to 7-fold greater than that of RsfabG1 under all conditions, and the level of expression of RsfabG2 under high salt concentration or low $\mathrm{pH}$ was almost same as that under natural conditions (Fig. 5d). These data indicate that the expression of RsfabG1 and RsfabG2 seems to be constitutive. We also complemented RS-G2 (RsfabG2::Gm) with plasmid pYJ35, which carries E. coli wild type EcfabG. This strain grew as well as strain GMI1000 under these conditions (Fig. 5). Therefore, we speculate that it is the level of activity of 3-ketoacyl-ACP reductase rather than the RsfabG2 gene or protein product that is important for the response of $R$. solanacearum to stress. This was also observed in the pathogenesis experiments described below.

\section{Mutation of RsfabG2 impaired the virulence of $R$. solanacearum in tomato plants}

$R$. solanacearum is a phytopathogenic bacterium that causes vascular wilt diseases in many plants [18]. To investigate the role of RsfabG2 in the virulence of $R$. solanacearum, tomato plants were inoculated with the wild type GMI1000 and the Rs-G2 (RsfabG2::Gm) strain. The wild type GMI1000 strain caused symptoms of wilt on the 3rd day after inoculation and the test tomato plants showed complete wilting by 7 days after infection, whereas the RS-G2 (RsfabG2::Gm) strain caused no disease symptoms until 21 days after inoculation (Fig. 6a). Furthermore, both RsfabG2 and EcfabG caused some tomato plant wilting and hence partially restored the virulence of the RS-G2 (RsfabG2::Gm) strain; restoration of EcfabG was more effective than that of RsfabG2. The mortality rates of tomato plants infected by these strains throughout the course of the infection were: GMI1000, 100 \%; RS-G2 (RsfabG2::Gm), 0 \%; RS-G2/pYJ34, $40 \%$ and RS-G2/pYJ35, $65 \%$.

We also evaluated several pathogenicity-related phenotypes of strain RS-G2 (RsfabG2::Gm). The production of EPS I (extracellular polysaccharide I), EGL (endoglucanase), and PGL (polygalacturonase) by RS-G2 (RsfabG2::Gm) was first tested. The data showed that production of EPS I, EGL, and PGL was not significantly impaired in the mutated strain RS-G2 (RsfabG2::Gm) (data not shown). Next, the biofilm formation and swimming motility of strain RSG2 (RsfabG2::Gm) were examined. After $R$. solanacearum strains had been incubated without shaking in BG broth at $28{ }^{\circ} \mathrm{C}$ for $36 \mathrm{~h}$, the biofilms formed on the slides were quantified. It was found that RS-G2 (RsfabG2::Gm) produced

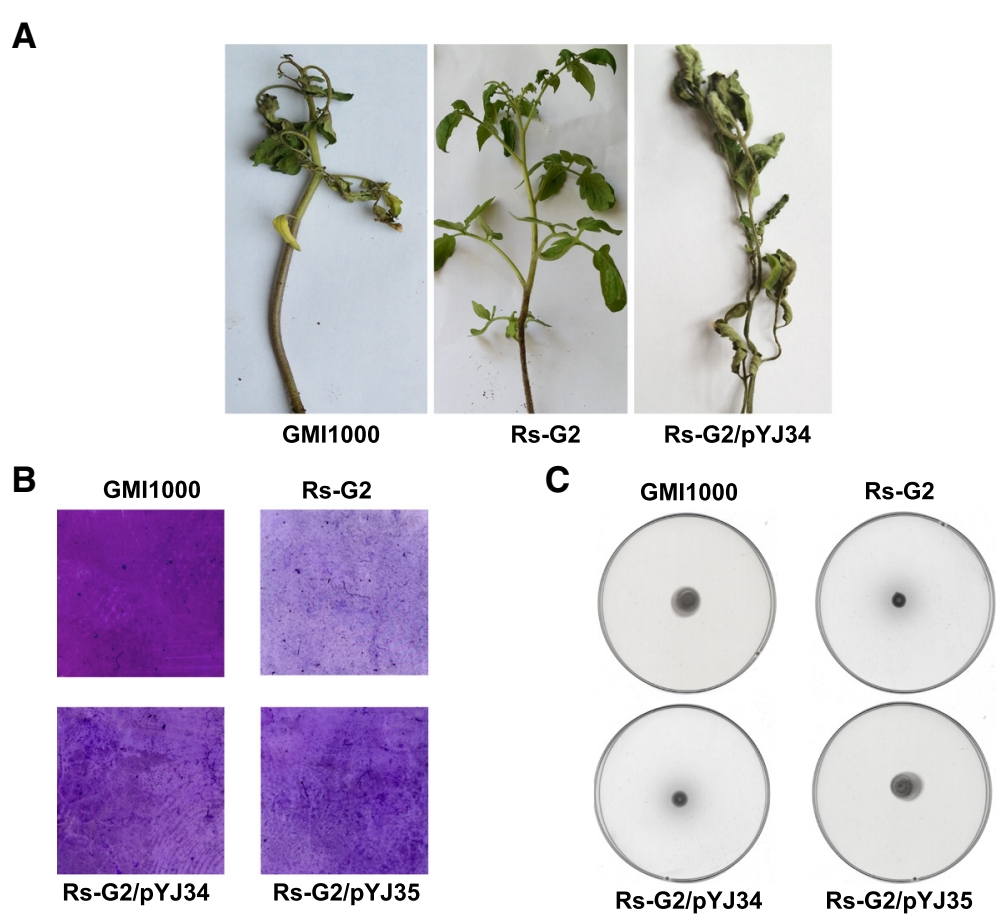

Fig. 6 Effects of RsfabG2 on virulence of R. solanacearum. Panel a, Pathogenicity test on tomato plants with the R. solanacearum GMI1000 or RS-G2 mutant strain. Panel b, Biofilm formation of strain RS-G2. Panel c, Swimming motility of the RS-G2 strain 
less biofilm $\left(\mathrm{OD}_{530}, 0.02 \pm 0.01\right)$ than the wild type strain GMI1000 ( $\left.\mathrm{OD}_{530}, 0.11 \pm 0.02\right)$ (Fig. 6b). The swimming motility of RS-G2 (RsfabG2::Gm) was evaluated on semisolid motility agar. After 2 days at $28{ }^{\circ} \mathrm{C}$, the RS-G2 (RsfabG2::Gm) swimming haloes were about half as large as those formed by GMI1000. When complemented with $E c f a b G$, the motility of the RS-G2 (RsfabG2::Gm) strain was restored to that of the wild type, whereas strain RS-G2 (RsfabG2::Gm) complemented with RsfabG2 formed the same small halo as RS-G2 (RsfabG2::Gm) (Fig. 6c). In order to explain why complementation of RS-G2 (RsfabG2::Gm) with RsfabG2 failed to restore swimming motility, the 3ketoacyl-ACP reductase activities were tested in cell-free extracts of these strains. Although the 3-ketoacyl-ACP reductase activity (in $\mu \mathrm{mol} / \mathrm{min}$ per $\mathrm{mg}$ extract protein) in strain RS-G2 carrying the RsfabG2 encoded plasmid $(0.375 \pm 0.015)$ was higher than that in strain RS-G2 $(0.250 \pm 0.068)$, it was still lower than that in strain RSG2/pYJ35 $(0.485 \pm 0.058)$ or the wild type strain $(0.576 \pm$ 0.056). These results indicate that the level of activity of 3-ketoacyl-ACP reductase is important for the lifestyle of $R$. solanacearum and that mutation of RsfabG2 can impair biofilm formation and decrease swimming motility.

To address the above observations further, we examined the expression of the genes related to $R$. solanacearum virulence determinants, including epsA, egl, pehB, pglA, fliC, fliM, pilQ, pilT and T3SS-related genes (popA, popC, hrcT, hrpK, hrpY, hrpX and $h r p V$ ), in the RS-G2(RsfabG2::Gm) mutant by quantitative RT-PCR. The expression of fliM, $h r p Y$, and $h r p X$ was obviously decreased in the RS-G2 (RsfabG2::Gm) mutant compared with the wild type strain GMI1000 ( $<<0.001)$ (Fig. 7a), whereas the level of expression of the remaining virulence-related genes in RS-G2 (RsfabG2::Gm) was not significantly different from that in the wild type strain $(\mathrm{p}>0.05)$ (data not shown). We also determined the levels of expression of six genes ( $h r p B, h r p G, p r h J$, prhR, prhI and $\operatorname{prh} A$ ) in a multigene regulatory cascade responsible for transcription of the entire $h r p$ regulon. When compared with the wild type strain GMI1000, the results showed that the expression level of $p r h A$ and $h r p G$ was significantly reduced in the RS-G2 (RsfabG2::Gm) mutant $(\mathrm{p}<0.001)$, and the expression of prhJ and $h r p B$ was slightly lower in the RS-G2 (RsfabG2::Gm) mutant than in GMI1000, while the level of prhR and prhI in RS-G2 (RsfabG2::Gm) was almost the same as in the wild type strain ( $\mathrm{p}>0.05$ ) (Fig. 7b). Meanwhile, both the RsfabG2 and the EcfabG genes could restore the expression of these genes in RS-G2 (RsfabG2::Gm) to the level of the wild type strain. These data indicated that impairment of $R$. solanacearum virulence by deletion of RsfabG2 seems to be due to reduction of the expression of various genes that are related to virulence determinants.
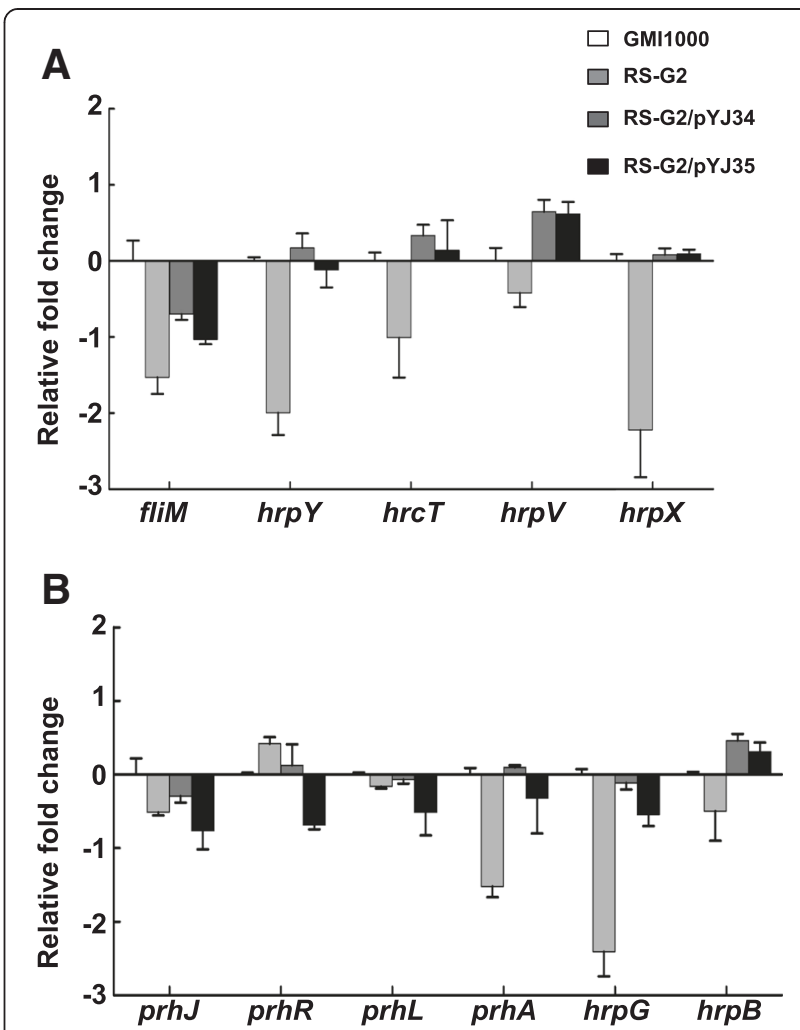

Fig. 7 Expression of the virulence-related genes in the RS-G2 mutant. Panel $\mathbf{a}$, Expression of the structural genes, including flim, hrpY, hrcT, hrpV and hrpX, in the RS-G2 strain. Panel $\mathbf{b}$, Expression of the regulator genes, including prhJ, prhR, prhl, prhA, hrpG and hrpB. Bacteria were cultured and RNA was extracted as described in the "Materials and Medthods". The rp/M gene was used as the internal control for quantitative RT-PCR. The experiment was performed at least three time using independent batches of samples with similar results. Results from a single representative sample are shown. The means \pm SD (error bars) of three determinations from CDNA from a single representative sample are shown

\section{Discussion}

In bacterial fatty acid synthesis, 3-ketoacyl-ACP reductase (KAR) catalyzes the key reduction of 3-ketoacylACPs to 3-hydroxy acyl-ACPs [3]. In E. coli, fabG is the only gene that encodes 3-ketoacyl-ACP reductase activity $[21,22]$. However, in other species of bacteria, multiple $f a b G$ paralogues have been annotated. For example, the genome of Mycobacterium tuberculosis has five annotated fabG genes [24]. Although M. tuberculosis fabG1 and fabG4 both complement a yeast $\Delta$ oar1 mutant strain (which lacks the mitochondrial 3-KAR activity), there is no other evidence for a bacterium that has more than one functional KAR. It should be noted that genomes generally encode many SDR family members and thus, in the absence of a genomic context consistent with a role in fatty acid synthesis, a true KAR can be very difficult to distinguish from other SDR family 
members catalyzing unrelated reactions, such as reduction of the keto group of an intermediate in sugar utilization. Moreover, even when a plausible genomic context is present, misannotation can occur, as was the case in Lactococcus lactis where a putative KAR lacked activity both in vivo and in vitro [17].

Growth of $R$. solanacearum requires RsFabG1, but not RsFabG2. The RsfabG1 gene is found within a highly conserved chromosomal gene cluster that apparently encodes several enzymes required for fatty acid synthesis, whereas the RsfabG2 gene is located on the R. solanacearum megaplasmid within a gene cluster containing $f a b B, f a b F 2$ and $f a b F 3$ [18]. Our prior studies have shown that the protein products encoded by the megaplasmid genes $f a b B, f a b F 2$ and $f a b F 3$ do not play roles in fatty acid synthesis [25], but we found that RsfabG2 complemented growth of the E. coli fabG(ts) strain CL104 and catalyzed all of the keto reduction steps in the elongation cycle of FAS II in vitro. Moreover, the strain in which RsfabG2 had been disrupted remained viable, although the mutant strain contained significantly more UFAs than the wild type strain, and was sensitive to a high salt concentration and low $\mathrm{pH}$. This result indicated that the RsfabG2 gene plays a role in growth rate and in adaptation to environmental stress. Furthermore, deletion of RsfabG2 reduced the ability of $R$. solanacearum to form a biofilm and impaired $R$. solanacearum swimming motility and the pathogenesis of disease in tomato plants. It should be noted that the $R$. solanacearum megaplasmid could reasonably be considered a second chromosome because a number of genes of primary metabolic pathways, including amino acid and cofactor biosynthesis, are encoded on the megaplasmid [18]. The megaplasmid also carries all of the hrp genes required to cause plant disease and encodes the constituents of the flagellum and most of the genes governing exopolysaccharide synthesis. This bipartite genome structure is characteristic of most $R$. solanacearum strains [26], and derivatives of strain GMI1000 lacking the megaplasmid have not been obtained [18]. Finally bioinformatic analyses indicate that the two replicons have shared a similar evolutionary history, suggesting that the megaplasmid was not recently acquired from another organism by lateral gene transfer but is rather a part of an ancestral $R$. solanacearum chromosome [27]. Therefore, the presence of a megaplasmid gene encoding a functional fatty acid synthetic enzyme and playing a role in virulence in tomato plants does not seem atypical, although this raises the question of the functions of the gene cluster (fabF3 RSp0360 fabG2 fabF2 fabB).

Although the protein products encoded by $f a b B, f a b F 2$ and $f a b F 3$ do not play roles in fatty acid synthesis, FabB, FabF2 and FabF3 indeed display higher identities with $E$. coli FabF, one of the long-chain 3-ketoacyl-ACP synthases [25]. Together with our observation about RsFabG2 in this report, we speculate that the gene products of this cluster ( $f a b F 3$ RSp0360 fabG2fabF2 $f a b B$ ) may constitute a novel pathway to synthesize an unidentified signal that is related to fatty acids, which may induce $R$. solanacearum pathogenesis in plants. A similar scenario has been investigated in Sinorhizobium meliloti, in which the nodFEG gene cluster was essential for the production of unusual $\alpha, \beta$ unsaturated fatty acid moieties of the Nod factors that trigger nodule formation on the roots of alfalfa. The gene products of nodF, nodE and nodG have recognizable homologies to acyl carrier protein (ACP), 3-ketoacyl-ACP synthase and 3-ketoacyl-ACP reductase, respectively. Therefore, if the enzymes encoded by the gene cluster (fabF3 RSp0360 fabG2fabF2 $f a b B$ ) are required to synthesize a specific signal that induces $R$. solanacearum pathogenesis in plants, it will be easy to understand why deletion of RsfabG2 from the megaplasmid caused the expression of virulence-related genes to decrease and reduced $R$. solanacearum pathogenesis in tomato plants.

Meanwhile, although both RsfabG2 and EcfabG partially complemented the $R$. solanacearum fabG2 mutation, EcfabG restored it more effectively than RsfabG2 itself. This suggested that the overall cellular level of 3ketoacyl-ACP reductase activity is important for the $R$. solanacearum lifestyle. Rhizobia present a similar scenario. The nodG gene is located in the nodFEG operon on the symbiotic plasmid of many rhizobia. It has been demonstrated that, although the NodG of $R$. leguminosarum has 3-ketoacyl-ACP reductase activity in vitro [28], the fabG gene cannot be deleted from the chromosome of S. meliloti. However, our experiments showed that, upon overexpression of nodG from plasmid pSRK-Gm, the fabG gene could be disrupted and the mutant strain was not defective in growth and fatty acid synthesis (data not published). This means that a high level of activity of 3-ketoacyl-ACP reductase is also essential to the growth of S. meliloti.

We also tried to disrupt RsfabG1 in strains where RsfabG2 was overexpressed from a plasmid, but our attempts were unsuccessful. Although the reason for this failure is unknown, it suggests that RsFabG2 and RsFabG1 are designed for distinct physiological roles. Although the catalytically active SDR triad, the $\mathrm{N}$-terminal cofactor binding sequence $[16,19,20]$, and two Arg residues important in binding the ACP moiety of the physiological substrate [21] are conserved in the two proteins (Fig. 1c), RsFabG2 is only $41 \%$ identical to RsFabG1. Moreover, RsFabG1 reduced acetoacetyl-CoA whereas RsFabG2 could not. We investigated whether poor expression of RsfabG2 explains its inability to bypass loss of RsfabG1. This does not appear to be the case, because RsfabG2 transcript levels were 6- to 7-fold greater than those of RsfabG1 in both log phase and stationary phase cultures (data not shown). 


\section{Conclusion}

In the bacterial fatty acid synthesis pathways studied to date only a single enzyme, FabG, has been found to catalyze the reduction of 3-ketoacyl-ACPs to 3-hydroxy acyl-ACPs. However, in this report we supply the first evidence that $R$. solanacearum encodes two functional FabG homologues. RsfabG1 is essential for growth of $R$. solanacearum, whereas RsfabG2 plays roles in determining the cellular fatty acid composition, adaptation to two environmental stresses and in the pathogenesis of disease in tomato plants. Therefore, like other enzymes in the bacterial fatty acid biosynthetic pathway, one bacterium may simultaneously possess two or more 3-oxoacyl-ACP reductase isozymes that have functions in fatty acid synthesis.

\section{Methods}

\section{Bacterial strains, plasmids and growth media}

The E. coli K-12 strains, $R$. solanacearum strains, and plasmids used in this study are listed in Additional file 4: Table S1. The $R$. solanacearum strains were routinely grown at $30{ }^{\circ} \mathrm{C}$ in $\mathrm{BG}$ broth or on BG agar (BG plus $1.6 \%$ agar) [25]. Luria-Bertani (LB) medium was used as the rich medium for $E$. coli. The phenotypes of $E$. coli fab strains were assessed on rich broth (RB) medium [29]. M63 medium [30] supplemented with $0.1 \%$ Casamino Acids was used to screen $R$. solanacearum mutants and, if needed, 5 or $10 \%$ sucrose was added. Antibiotics were used at the following concentrations (in $\mu \mathrm{g} / \mathrm{ml}$ ): sodium ampicillin, 100; kanamycin sulfate, 30; chloramphenicol, 30; and gentamicin sulfate, 10 (for E. coli) or 30 (for $R$. solanacearum). L-Arabinose was used at a final concentration of $0.01 \%$. Isopropyl- $\beta$-D-thiogalactoside (IPTG) was used at a final concentration of $1 \mathrm{mM}$, and 5-bromo-4chloro-3-indolyl- $\beta$ - $\mathrm{D}$-galactoside (X-Gal) was used at a final concentration of $20 \mu \mathrm{g} / \mathrm{ml}$.

\section{Recombinant DNA techniques and construction of plasmids}

To obtain the $R$. solanacearum fabG genes, genomic DNA was extracted from strain GMI1000 using the Takara DNA extraction kit. The genomic DNA was then used for PCR amplification with Pfu DNA polymerase and the primers listed in Additional file 1: Table S2, and the products were inserted into T-vector plasmid pMD19 to give plasmids pYJ1 (RsfabG1) and pYJ2 (RsfabG2). The fabG sequences were confirmed by sequencing done by Shanhai Sangon, Inc. To produce plasmids pYJ3 (RsfabG1), pYJ4 (RsfabG2), pYJ5 (RsfabG1) and pYJ6 (RsfabG2), the T-vector pMD19 fab gene plasmids were digested with NdeI and HindIII. The fragments were gel purified and ligated into either pBAD24M [11] or pET$28 \mathrm{~b}$ digested with the same enzymes. The NdeI-HindIII fragments from pYJ1 (RsfabG1) and pYJ2 (RsfabG2) were ligated into pSRK-Tc or pSRK-Km digested with the same enzymes to yield pYJ33 (RsfabG1) and pYJ34 (RsfabG2), respectively. Meanwhile, we also constructed pYJ35, in which E. coli fabG was inserted into NdeI and HindII sites of pSRK-Km.

\section{Disruption and essentiality testing of the RsfabG genes}

To disrupt $R$. solanacearum fabG1, a suicide plasmid was constructed as follows. The 500 bp DNA fragments located upstream and downstream of RsfabG1 (called Up fabG1 and Down fabG1, respectively) were amplified with $P f u$ DNA polymerase using $R$. solanacearum genome DNA as the template and either RsFabG1 Knt up EcoRI and RsFabG1 Knt up 2 (for Up fabG1) or RsFabG1 Knt down 1 and RsFabG1 Knt down HindIII (for Down fabG1) as primers (Additional file 1: Table S2). The products of these PCR reactions were purified, and overlap PCR was carried out using RsFabG1 Knt up EcoRI and RsFabG1 Knt down HindIII as the primers. The resulting 1000 bp DNA fragment was digested with EcoRI and HindIII and inserted between the same sites of pHSG399 [31] to yield pYJ25. A 500 bp gentamicin resistance cassette was also amplified from plasmid p34s-Gm [32] with Gm up BspHI plus Gm down XbaI (Additional file 1: Table S2) as the primers. The PCR product was digested with $\mathrm{BspHI}$ and $\mathrm{XbaI}$ and cloned between the same sites of pYJ25 to give pYJ26. The 1500 bp fabG1:: Gm fragment of pYJ26 was digested with EcoRI and HindIII and ligated into the same sites of pK18mobsacB [33] to yield pYJ27. In the same manner we constructed pYJ30, which carried a 1500 bp fabG2:: Gm fragment. To replace $R$. solanacearum fabG1 with E. coli CL104 fabG (ts) [22], a suicide plasmid was constructed as follows. The 750 bp EcfabG gene was amplified with $P f u$ DNA polymerase using $E$. coli CL104 genomic DNA as the template and EcFabG (ts) up and EcFabG (ts) down as primers. A 750 bp gentamicin resistance cassette was also amplified from plasmid p34sGm with Gmdp up plus Gmdp down Xbal (Additional file 1: Table S2) as the primers. The products of these PCRs were purified, and overlapping PCR was carried out using EcFabG (ts) up and Gmdp down XbaI as the primers. The 1500 bp DNA fragment containing EcfabG-Gm was digested with $\mathrm{BspHI}$ and $\mathrm{XbaI}$ and inserted into the same sites of pYJ25 to yield pYJ31. The 2500 bp PCR fragment containing the RsfabG1::[ $\Phi(E c f a b G-\mathrm{Gm}]$ digested with EcoRI and HindIII from pYJ31 was inserted into pK18mobsacB to yield pYJ32.

A derivative of $E$. coli strain S17-1 carrying plasmids pYJ27 (RsfabG1), pYJ30 (RsfabG2) or pYJ32 (EcfabG) was mated with $R$. solanacearum GMI1000 on BG plates for $24 \mathrm{~h}$ at $30{ }^{\circ} \mathrm{C}$. The cells were suspended in BG medium and appropriate dilutions were spread on BG plates containing chloramphenicol (to select against the donor strain) plus gentamicin and kanamycin to select 
for integration of the non-replicating plasmid into the genome of the recipient. Several colonies were inoculated into BG medium, and the cultures were incubated at $30{ }^{\circ} \mathrm{C}$ for $24 \mathrm{~h}$, after appropriate dilutions were spread on BG plates containing $10 \%$ sucrose. The resulting colonies were inoculated onto BG plates containing kanamycin or gentamicin using sterile toothpicks. Colonies resistant to gentamicin and sensitive to kanamycin were screened by colony PCR utilizing the primers listed in Additional file 1: Table S2. RS-G2, the RsfabG2 disruption mutant, and RS-G3, the $R$. solanacearum strain in which RsfabG1 was replaced by E. coli fabG(ts), were obtained. We failed to obtain a RsfabG1 deletion strain; only RsfabG1 merodiploids were obtained.

To disrupt the RsfabG1 gene from the $R$. solanacearum GMI1000 chromosome, plasmid pYJ33 was introduced into the RsfabG1 merodiploid. After selection on BG plates containing $10 \%$ sucrose, a mutant strain RS-G5 was obtained.

\section{Expression and purification of plasmid-encoded proteins} The pET28b-derived plasmids carrying RsfabG alleles were introduced into E. coli strain BL21 (DE3), and the respective proteins, RsFabG1and RsFabG2, were expressed at high levels and purified as described previously $[11,13]$. The enzymes were homogeneous as judged by SDSPAGE. The E. coli FabD, FabH, FabG, FabZ, and FabI, Vibrio harveyi AasS and E. coli holo-ACP proteins were purified as described previously $[11,13]$.

The solution structures of RsFabG1 and RsFabG2 were analyzed by size exclusion chromatography on a Superdex 75 10/300 GL column (GE Healthcare) using an Äkta fast protein liquid chromatography (FPLC) system (Pharmacia) at $0.45 \mathrm{ml} / \mathrm{min}$ in phosphate running buffer $\left(135 \mathrm{mM} \mathrm{NaCl}, 2.7 \mathrm{mM} \mathrm{KCl}, 1.5 \mathrm{mM} \mathrm{Na}_{2} \mathrm{HPO}_{4}\right.$ and $8 \mathrm{mM} \mathrm{K}_{2} \mathrm{HPO}_{4}, 10 \%$ glycerol, pH 7.4) and the standards used previously $[13,34]$.

\section{Circular dichroism measurements}

The circular dichroism (CD) spectra of FabGs were obtained on the Chirascan (Applied Photophysics Limited, Leatherhead, Surrey, UK) at $25{ }^{\circ} \mathrm{C}$ using a $1.0 \mathrm{~nm}$ bandwidth, $1 \mathrm{~mm}$ cell, $1.0 \mathrm{~nm}$ step, 0.5 dwell time and 1.0 min time internal. The CD spectra were measured at an enzyme concentration of $4 \mu \mathrm{M}$ in $50 \mathrm{mM}$ sodium phosphate buffer ( $\mathrm{pH} 7.5$ ). The results were expressed as molar ellipticity $(\theta) \mathrm{deg} \mathrm{cm}^{2} \mathrm{dmol}^{-1}$. The values were normalized by subtracting the baseline recorded for the buffer under similar conditions.

\section{Assay of RsFabG1 and RsFabG2 activities in vitro}

Malonyl-ACP was synthesized from holo-ACP and malonyl-CoA using E. coli FabD; octanoyl-ACP, tetradecanoyl-ACP, 3-hydroxydecanoyl-ACP and 3-
hydroxyhexadecanoyl-ACP were synthesized from the acids, ATP and E. coli holo-ACP by $V$. harveyi acylACP synthetase as described previously [35]. The abilities of RsFabG1 and RsFabG2 to function in the first cycle of fatty acid synthesis were assessed in reaction mixtures containing $0.1 \mathrm{M}$ sodium phosphate $(\mathrm{pH} 7.0) ; 0.1 \mu \mathrm{g}$ each of EcFabH, EcFabZ and EcFabI; $50 \mu \mathrm{M}$ NADH; $50 \mu \mathrm{M}$ NADPH; 1 mM-mercaptoethanol; $100 \mu \mathrm{M}$ acetyl-CoA; $50 \mu \mathrm{M}$ malonyl-ACP; and $50 \mu \mathrm{M}$ holo-ACP in a final volume of $40 \mu \mathrm{l}$. To investigate the reduction of long-chain 3-ketoacyl-ACP, the reaction mixtures contained $0.1 \mathrm{M}$ sodium phosphate ( $\mathrm{pH} 7.0$ ); $50 \mu \mathrm{M}$ malonyl-ACP; $50 \mu \mathrm{M}$ long chain acyl-ACP (octanoyl-ACP or tetradecanoyl-ACP); $0.1 \mu \mathrm{g}$ each of EcFabB and EcFabZ; and $50 \mu \mathrm{M}$ $\mathrm{NADPH}$. The reactions were initiated by the addition of $0.1 \mu \mathrm{g}$ KAR (3-ketoacyl-ACP reductase) (EcFabG, RsFabG1 or RsFabG2), followed by incubation for $1 \mathrm{~h}$ at $37{ }^{\circ} \mathrm{C}$. The reaction products were resolved by conformationally sensitive gel electrophoresis on $17.5 \%$ polyacrylamide gels containing a concentration of urea optimized for the separation [11]. The gel was stained with Coomassie Brilliant Blue R250.

\section{NADH oxidation assay}

The 3-ketoacyl-ACP reductase activity was monitored using the decrease in absorbance at $340 \mathrm{~nm}$ by UV-vis spectrophotometry with an NADPH extinction coefficient of $6220 \mathrm{Mol}^{-1}$. Each $500 \mu \mathrm{l}$ reaction was performed in UV-transparent microcuvettes. The activity assays contained varying concentrations of NADPH, $0.1 \mu \mathrm{g}$ of the purified native $R$. solanacearum FabG, varying substrate concentrations of acetoacetyl-CoA, and $0.1 \mathrm{M}$ $\mathrm{LiCl}$ in a $0.1 \mathrm{M}$ sodium phosphate buffer ( $\mathrm{pH} 7$ ). Kinetic constants were determined using GraphPad PRISM version 4 software. The Km values for NADPH were determined at an acetoacetyl-CoA concentration of $200 \mu \mathrm{M}$. The $\mathrm{Km}$ values for acetoacetyl-CoA were determined using $200 \mu \mathrm{M}$ NADPH.

\section{Analysis of fatty acid compositions}

The cellular lipid assay was adapted from that of Stead [36]. Briefly, cultures were grown on BG agar or M63 agar. Cells were harvested from plates with a sterile aluminum spatula into small screw-capped test tubes. Cellular lipids were saponified by addition of $1 \mathrm{ml}$ $\mathrm{NaOH}$ in methanol solution $(\mathrm{NaOH} 45$ g; methanol $150 \mathrm{ml}$; water $150 \mathrm{ml}$ ). The samples were placed in a boiling water bath for $30 \mathrm{~min}$. The tubes were vortexed before and once during boiling. Fatty acids were methylated by addition of $2 \mathrm{ml} 6 \mathrm{M} \mathrm{HCl}$ in methanol $(325 \mathrm{ml}$ $11.6 \mathrm{M} \mathrm{HCl}$, plus $275 \mathrm{ml}$ methanol). The samples were heated at $80{ }^{\circ} \mathrm{C}$ for $10 \mathrm{~min}$ and immediately cooled to below $20^{\circ} \mathrm{C}$. The fatty acid methyl esters were extracted 
three times with $1.25 \mathrm{ml}$ petroleum ether. The samples were dried under a stream of nitrogen in a fume hood. The esters were analyzed by gas chromatography-mass spectrometry (GC-MS) as described previously [37]. The data are presented as percentages of the total fatty acids and represent the means \pm standard error for three independent determinations.

\section{Pathogenicity tests}

Pathogenicity tests on soil-grown plants were conducted as already described [38]. Briefly, healthy 19- to 21-dayold plants were inoculated by pouring a bacterial suspension onto the soil to a final density of approximately $6 \times 10^{8} \mathrm{CFU} / \mathrm{g}$ soil, followed by incubation at $28{ }^{\circ} \mathrm{C}$. Each strain tested was assessed for wilting in three independent 10-plant experiments.

\section{Motility assays}

The motility of wild type and mutant strains was assayed on semisolid motility media containing $1 \%$ (wt/vol) tryptone and $0.325 \%$ (wt/vol) noble agar [39]. The plates were inoculated with a $2-\mu \mathrm{l}$ drop of bacterial culture containing $1 \times 10^{6} \mathrm{CFU} / \mathrm{ml}$. Motility was visualized as a white halo of cells moving outward from the original inoculation site after 3 to 5 days of incubation at $28{ }^{\circ} \mathrm{C}$.

\section{Biofilm assay}

To quantify biofilm formation, we used a tube biofilm assay. Briefly, $0.2 \mathrm{ml}$ of 2-day cultures of $R$. solanacearum were inoculated into $20 \mathrm{~mL}$ fresh BG broth in a 50 -mL tube. A cover slide (microscope cover glass, $15 \times$ $15 \mathrm{~mm}$, sterilized with $70 \%$ ethanol) was put into the tube. The culture was incubated without shaking for $36 \mathrm{~h}$ at $28{ }^{\circ} \mathrm{C}$. Crystal violet staining and biofilm quantification by absorbance at $530 \mathrm{~nm}$ were performed as described previously [40].

\section{RNA extractions and quantitative real-time PCR analyses}

The $R$. solanacearum wild type strain or its mutants were cultured using M63 medium containing $20 \mathrm{mM}$ glutamate as previously described [41]. The total RNA of $R$. solanacearum was extracted using a TRIzol ${ }^{\circ} \mathrm{Max}^{\mathrm{Tm}}$ Bacterial RNA Isolation Kit (Thermo Scientific). The RNAs were adjusted to a concentration of $200-500 \mathrm{ng} / \mu \mathrm{l}$ as measured with a NanoDrop 8000 (Thermo Scientific), and all samples were reverse-transcribed using a PrimeScript ${ }^{\mathrm{mi}}$ RT Reagent Kit with gDNA Eraser (Clontech). Quantitative real-time PCR analyses were carried out on an Applied Biosystems PRISM model 7500 Sequence Detection system with Maxima ${ }^{\circ}$ SYBR Green qPCR Master Mix (Thermo Scientific). Relative quantitation was done by the comparative cycle threshold method using the endogenous internal control rplM for sample normalization as previously described [42]. The amplification program was as follows: $10 \mathrm{~min}$ at $95{ }^{\circ} \mathrm{C}$;
40 cycles of $95{ }^{\circ} \mathrm{C}$ for $15 \mathrm{~s}, 57^{\circ} \mathrm{C}$ for $1 \mathrm{~min}$. The oligonucleotides used as primers are indicated in Additional file 5: Table S3. Three independent experiments were carried out for each strain and three technical replicate reactions of RT-PCR were used for each sample.

\section{Ethics statement}

As no human or animal subjects were used for this work, consent and ethical approval were not required.

\section{Additional file}

Additional file 1: Table S2. Sequences of the PCR primers used in this work.

Additional file 2: Figure S1. Strategy for isolation R. solanacearum fabGs mutant strains.

Additional file 3: Figure S2. PCR analysis of the genomic DNAs of R. solanacearum fabG1 and fabG2 mutant strains.

Additional file 4: Table S1. Strains and plasmids used in this work. Additional file 5: Table S3. Sequences of the RT-PCR primers used in this work.

\section{Abbreviations}

SDR: Short chain dehydrogenase/reductase; FAS: Fatty acid synthase; ACP: Acyl carrier protein; KAR: 3-ketoacyl-acyl carrier protein reductase; FabD: Malonyl-CoA:ACP transacylase; FabH: 3-ketoacyl-ACP synthase III; FabG: 3-ketoacyl-ACP reductase; FabZ: 3-hydroxyacyl-ACP dehydrase; Fabl: Enoyl-ACP reductase; AasS: Acyl-ACP synthetase; CD: Circular dichroism; Km: Kanamycin; Gm: Gentamicin; Amp: Sodium ampicillin; Cm: Chloramphenicol; GC-MS: Gas chromatography-mass spectrometry; FA: Fatty acid; UFA: Unsaturated fatty acid; SFA: Saturated fatty acid; HFA: 3-hydroxy fatty acid; EPS I: Extracellular polysaccharide I;

PGL: Polygalacturonase; EGL: Endoglucanase; IPTG: Isopropyl $\beta-D-$ Thiogalactoside; X-Gal: 5-bromo-4-chloro-3-indolyl- $\beta$-D-galactoside; n-C14:0: tetradecanoic acid; n-C14:0 3-OH: 3-hydroxyl-tetradecanoic acid; $\mathrm{n}-\mathrm{C} 16: 1$ cis 9: cis-9-hexadencenoic acid; n-C16:0: Hexadecanoic acid; n-C16:0 2-OH: 2-hydroxyl-hexadencenoic acid; n-C17:0 cyclo: cis-9,10-methylene hexdecanoic acid; $n-C 18: 1$ cis 11: cis-11-octadencenoic acid; n-

C18:0: Octadencenoic acid.

\section{Competing interests}

The authors declare that they have no competing interests.

\section{Authors' contributions}

SX cloned the R. solanacearum fabGs genes, constructed several fabG expression vectors, did complementation experiments with fabGs expression vectors, purified FabGs protein and carried out biochemical studies on FabGs in vitro. JY constructed fabGs mutants and tested the growth of mutants in BG medium. JC and ZH carried out experiments on the pathogenesis of $R$. solanacearum in tomato plants. LZ helped with size exclusion chromatography and CD measurements. HK and YS helped to design the PCR primers and carried out GRT-PCR. HW conceived of the study, and participated in its design and coordination and helped to draft the manuscript. All authors read and approved the final manuscript.

\section{Acknowledgments}

This work was supported by grants from the National Key Project for Basic Research (2015CB150600), the National Natural Science Foundation of China (31471743 and 31200028), and the Specialized Research Fund for the Doctoral Program of Higher Education of China (20104404110005). The authors are grateful to Prof. John E. Cronan in UIUC for his constructive revision of our manuscript. 


\section{Author details}

${ }^{1}$ Guangdong Provincial Key Laboratory of Protein Function and Regulation in Agricultural Organisms, College of Life Sciences, South China Agricultural University, No.483 Wushan Road, Tianhe, Guangzhou 510642, P. R. China. ${ }^{2}$ Departments of Microbiology and Biochemistry, University of Illinois at Urbana-Champaign, Urbana, IL 61801, USA. ${ }^{3}$ Department of Pathogenic Biology, Jiangsu Key Laboratory of Pathogenic Biology, Nanjing Medical University, Nanjing, Jiangsu 210029, China. ${ }^{4}$ Guangzhou Institutes of Biomedicine and Health, Chinese Academy of Sciences, Guangzhou, Guangdong 510530, China.

Received: 14 May 2015 Accepted: 8 October 2015

\section{Published online: 22 October 2015}

\section{References}

1. Chan DI, Vogel HJ. Current understanding of fatty acid biosynthesis and the acyl carrier protein. Biochem J. 2010;430:1-19.

2. Zhang YM, Rock CO. Membrane lipid homeostasis in bacteria. Nat Rev Microbiol. 2008;6(3):222-33.

3. White SW, Zheng J, Zhang YM, Rock CO. The structural biology of Type II fatty acid biosynthesis. Annu Rev Biochem. 2004;74:791-831.

4. Lu YJ, Zhang YM, Rock CO. Product diversity and regulation of type II fatty acid synthases. Biochem Cell Biol. 2004;82(1):145-55.

5. Heath RJ, White SW, Rock CO. Lipid biosynthesis as a target for antibacterial agents. Prog Lipid Res. 2001;40(6):467-97.

6. Campbell JW, Cronan Jr JE. Bacterial fatty acid biosynthesis: targets for antibacterial drug discovery. Annu Rev Microbiol. 2001;55:305-32.

7. Massengo-Tiasse RP, Cronan JE. Diversity in enoyl-acyl carrier protein reductases. Cell Mol Life Sci. 2009;66(9):1507-17.

8. Marrakchi H, Zhang YM, Rock CO. Mechanistic diversity and regulation of Type II fatty acid synthesis. Biochem Soc Trans. 2002;30(6):1050-5.

9. Wang $\mathrm{H}, \mathrm{Cronan} J \mathrm{E}$. Functional replacement of the FabA and FabB proteins of Escherichia coli fatty acid synthesis by Enterococcus faecalis FabZ and FabF homologues. J Biol Chem. 2004;279(33):34489-95.

10. Lu YJ, White SW, Rock CO. Domain swapping between Enterococcus faecalis FabN and FabZ proteins localizes the structural determinants for isomerase activity. J Biol Chem. 2005;280(34):30342-8.

11. Zhu L, Lin J, Ma J, Cronan JE, Wang H. Triclosan resistance of Pseudomonas aeruginosa PAO1 is due to FabV, a triclosan-resistant enoyl-acyl carrier protein reductase. Antimicrob Agents Chemother. 2010;54(2):689-98.

12. Heath RJ, Su N, Murphy CK, Rock CO. The enoyl-[acyl-carrier-protein] reductases Fabl and FabL from Bacillus subtilis. J Biol Chem. 2000;275(51):40128-33

13. Zhu L, Bi H, Ma J, Hu Z, Zhang W, Cronan JE, et al. The Two Functional Enoyl-Acyl Carrier Protein Reductases of Enterococcus faecalis Do Not Mediate Triclosan Resistance. mBio. 2013;4(5):e00613-13.

14. Marrakchi H, Dewolf Jr WE, Quinn C, West J, Polizzi BJ, So CY, et al. Characterization of Streptococcus pneumoniae enoyl-(acyl-carrier protein) reductase (FabK). Biochem J. 2003;370(Pt 3):1055-62.

15. Oppermann U, Filling C, Hult M, Shafqat N, Wu X, Lindh $M$, et al. Short-chain dehydrogenases/reductases (SDR): the 2002 update. Chem Biol Interact. 2003;143-144:247-53.

16. Price AC, Zhang YM, Rock CO, White SW. Structure of beta-ketoacyl-[acyl carrier protein] reductase from Escherichia coli: negative cooperativity and its structural basis. Biochemistry. 2001;40(43):12772-81.

17. Wang H, Cronan JE. Only one of the two annotated Lactococcus lactis fabG genes encodes a functional beta-ketoacyl-acyl carrier protein reductase. Biochemistry. 2004;43(37):11782-9.

18. Salanoubat M, Genin S, Artiguenave F, Gouzy J, Mangenot S, Arlat M, et al. Genome sequence of the plant pathogen Ralstonia solanacearum. Nature. 2002;415(6871):497-502.

19. Rafferty JB, Fisher M, Langridge SJ, Martindale W, Thomas NC, Simon JW, et al. Crystallization of the NADP-dependent beta-keto acyl carrier protein reductase from Escherichia coli. Acta Crystallogr D Biol Crystallogr. 1998;54(3):427-9.

20. Price AC, Zhang YM, Rock CO, White SW. Cofactor-induced conformational rearrangements establish a catalytically competent active site and a proton relay conduit in FabG. Structure. 2004;12(3):417-28.

21. Zhang YM, Wu B, Zheng J, Rock CO. Key residues responsible for acyl carrier protein and beta-ketoacyl-acyl carrier protein reductase (FabG) interaction. J Biol Chem. 2003;278(52):52935-43.
22. Lai CY, Cronan JE. Isolation and characterization of beta-ketoacyl-acyl carrier protein reductase ( $\mathrm{fabG}$ ) mutants of Escherichia coli and Salmonella enterica serovar Typhimurium. J Bacteriol. 2004;186(6):1869-78.

23. Heath RJ, Rock CO. Enoyl-acyl carrier protein reductase (fabl) plays a determinant role in completing cycles of fatty acid elongation in Escherichia coli. J Biol Chem. 1995;270(44):26538-42.

24. Gurvitz A. The essential mycobacterial genes, fabG1 and fabG4, encode 3-oxoacyl-thioester reductases that are functional in yeast mitochondrial fatty acid synthase type 2. Mol Genet Genomics. 2009;282(4):407-16.

25. Cheng J, Ma J, Lin J, Fan ZC, Cronan JE, Wang H. Only one of the five Ralstonia solanacearum long-chain 3-ketoacyl-acyl carrier protein synthase homologues functions in fatty acid synthesis. Appl Environ Microbiol. 2012;78(5):1563-73.

26. Rosenberg C, Casse-Delbart F, Dusha I, David M, Boucher C. Megaplasmids in the plant-associated bacteria Rhizobium meliloti and Pseudomonas solanacearum. J Bacteriol. 1982;150(1):402-6.

27. Coenye T, Vandamme P. Simple sequence repeats and compositional bias in the bipartite Ralstonia solanacearum GMl1000 genome. BMC Genomics. 2003;4(1):10.

28. Lopez-Lara IM, Geiger O. The nodulation protein NodG shows the enzymatic activity of an 3-oxoacyl-acyl carrier protein reductase. Mol Plant-Microbe Interac. 2001;14(3):349-57.

29. Ulrich AK, de Mendoza D, Garwin JL, Cronan Jr JE. Genetic and biochemical analyses of Escherichia coli mutants altered in the temperature-dependent regulation of membrane lipid composition. J Bacteriol. 1983;154(1):221-30.

30. Miller JH. A short course in bacterial genetics: a laboratory manual and handbook for Escherichia coli and related bacteria. Plainview, N.Y.: Cold Spring Harbor Laboratory Press; 1992.

31. Takeshita S, Sato M, Toba M, Masahashi W, Hashimoto-Gotoh T. High-copy-number and low-copy-number plasmid vectors for lacZ alpha-complementation and chloramphenicol- or kanamycin-resistance selection. Gene. 1987;61(1):63-74.

32. Dennis JJ, Zylstra GJ. Improved antibiotic-resistance cassettes through restriction site elimination using Pfu DNA polymerase PCR. BioTechniques. 1998;25(5):772-6.

33. Schafer A, Tauch A, Jager W, Kalinowski J, Thierbach G, Puhler A. Small mobilizable multi-purpose cloning vectors derived from the Escherichia coli plasmids pK18 and pK19: selection of defined deletions in the chromosome of Corynebacterium glutamicum. Gene. 1994;145(1):69-73.

34. Feng $Y$, Cronan JE. The Vibrio cholerae fatty acid regulatory protein, FadR, represses transcription of $p / s B$, the gene encoding the first enzyme of membrane phospholipid biosynthesis. Mol Microbiol. 2011;81(4):1020-33.

35. Jiang Y, Chan $\mathrm{CH}$, Cronan JE. The soluble acyl-acyl carrier protein synthetase of Vibrio harveyi B392 is a member of the medium chain acyl-CoA synthetase family. Biochemistry. 2006;45(33):10008-19.

36. Stead DE. Grouping of plant-pathogenic and some other Pseudomonas spp. by using cellular fatty acid profiles. Int J Syst Bacteriol. 1992;42:15.

37. Libia Saborido Basconcillo BEM. Comparison of three GC/MS methodologies for the analysis of fatty acids in Sinorhizobium meliloti: Development of a micro-scale, one-vial method. J Chromatogr B. 2008;871:22-31.

38. Tans-Kersten J, Guan Y, Allen C. Ralstonia solanacearum pectin methylesterase is required for growth on methylated pectin but not for bacterial wilt virulence. Appl Environ Microbiol. 1998;64(12):4918-23.

39. Yao J, Allen C. Chemotaxis is required for virulence and competitive fitness of the bacterial wilt pathogen Ralstonia solanacearum. J Bacteriol. 2006;188(10):3697-708.

40. Meng F, Yao J, Allen C. A MotN mutant of Ralstonia solanacearum is hypermotile and has reduced virulence. J Bacteriol. 2011;193(10):2477-86.

41. Yang WC, Lin YM, Cheng YS, Cheng CP. Ralstonia solanacearum RSC0411 (lptC) is a determinant for full virulence and has a strain-specific novel function in the T3SS activity. Microbiology. 2013;159(6):1136-48.

42. Monteiro F, Genin S, van Dijk I, Valls M. A luminescent reporter evidences active expression of Ralstonia solanacearum type III secretion system genes throughout plant infection. Microbiology. 2012;158(8):2107-16. 